\title{
ÜBER PRODUKTE VON NILPOTENTEN GRUPPEN
}

\author{
G. A. Miller zum Gedächtnis \\ VON \\ Helmut Wielandt \\ 1. Übersicht
}

Ein bekannter Satz von P. HALL [2, p. 198; 3, p. 319] besagt:

Eine endliche Gruppe ist genau dann auflösbar, wenn sie sich als das Produkt von paarweise vertauschbaren Untergruppen von Primzahlpotenzordnung darstellen läßt.

Dieser Satz ist deswegen von Bedeutung, weil er einen engen Zusammenhang zwischen der Normalstruktur und der arithmetischen Struktur herstellt. Die beiden in ihm enthaltenen Aussagen liegen verschieden tief. Der Nachweis, daß die Bedingung hinreicht, ist weit schwieriger als der der Notwendigkeit. Daher erscheint jede Abschwächung der hinreichenden Bedingung von Interesse. In dieser Richtung beweisen wir:

Satz 1. Die endliche Gruppe $G$ sei das Produkt von nilpotenten Untergruppen $G_{1}, \cdots, G_{r}$, von denen je zwei als Ganze vertauschbar sind und teilerfremde Ordnungen haben. Dann ist $G$ auflösbar.

Den Beweis brauchen wir nur für $r=2 \mathrm{zu}$ führen; denn es ist bekannt, daß das Produkt von mehreren paarweise vertauschbaren nilpotenten endlichen Gruppen genau dann auflösbar ist, wenn alle Produkte von je zweien dieser Gruppen auflösbar sind [5, p. 1]. Wir beweisen den Satz für $r=2$ in einer erweiterten Fassung:

SATz 2. Es sei $G=A B$; darin seien $A, B$ nilpotente Untergruppen von $G$, deren Ordnungen $|A|,|B|$ teilerfremd sind. Dann ist $G$ auflösbar; und wenn $P, Q$ Sylowgruppen von $G$ sind, derart daß $|P|$ ein Teiler von $|A|,|Q|$ ein Teiler von $|B|$ ist, so ist $P Q=Q P$.

Die Voraussetzung $G=A B$ kann durch $|G|=|A| \cdot|B|$ ersetzt werden, da $A$ und $B$ wegen der Teilerfremdheit der Ordnungen nur das Einselement 1 gemeinsam haben.

Es erscheint schwierig, die Voraussetzung der Nilpotenz noch weiter abzuschwächen. Keinesfalls genügt es für die Auflösbarkeit von $A B$, wenn $A$ nilpotent und $B$ überauflösbar ist; das sieht man aus der Zerlegung der Ikosaedergruppe in Faktoren der Ordnungen 5 und 12.

Offen bleibt die interessante Frage, ob man die Voraussetzung der Teilerfremdheit weglassen kann.

Received November 4, 1958. 
Der Beweis von Satz 2 beruht auf allgemein gültigen Betrachtungen über faktorisierte Gruppen (Abschnitt 2).

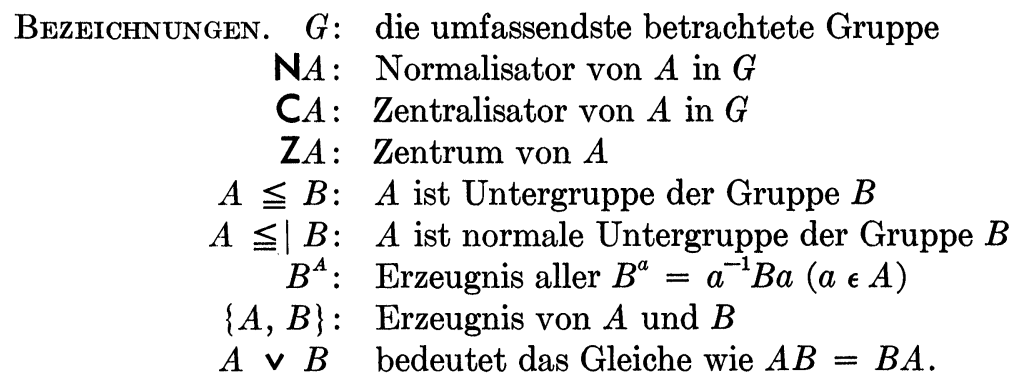

\section{Faktorisierte Gruppen}

In diesem Abschnitt sei $G$ eine Gruppe beliebiger, nicht notwendig endlicher Ordnung, und es sei eine bestimmte Zerlegung von $G$ in das Produkt von zwei Untergruppen gegeben: $G=A B$. Utber den Durchschnitt $A \cap B$ wird nichts vorausgesetzt. Wir wollen eine Untergruppe $F$ von $G$ kurz als faktorisiert bezeichnen, wenn

$$
F=(F \cap A)(F \cap B) \text { und } A \cap B \leqq F
$$

ist. Die ganze Gruppe $G$ ist faktorisiert, ebenso der Durchschnitt $A \cap B$. Offenbar gilt

Hilfssatz 3. Sei $F \leqq G$. Genau dann ist $F$ faktorisiert, wenn aus $a b^{-1} \epsilon F$, $a \in A, b \in B$ stets $a \in F$ folgt.

Hieraus liest man ab:

HILfssatz 4. Jede Obergruppe von $A$ ist faktorisiert; das Gleiche gilt für $B$.

Um nichttriviale faktorisierte Untergruppen zu finden, gehen wir von der folgenden Bemerkung aus:

Hilfssatz 5. Sei $H \leqq G$. Aus $a \in A, b \in B$ und $H^{a}=H^{b}$ folge stets $H^{a}=H^{b}=H$. Dann ist der Normalisator von $H$ in $G$ faktorisiert.

Beweis. Ist $a b^{-1} \epsilon \mathbf{N} H$, so ist $H^{a}=H^{b}$, also $H^{a}=H, a \epsilon \mathbf{N} H$.

Um Gruppen $H \mathrm{zu}$ finden, welche die Voraussetzung dieses Hilfssatzes erfüllen, benutzen wir die invarianten Relationen zwischen den Untergruppen von $G$. Damit meinen wir diejenigen Relationen $\mathbf{R}$, die bei allen inneren Automorphismen erhalten bleiben: Aus $C \leqq G, D \leqq G, C \mathrm{R} D$ und $g \in G$ folgt stets $C^{g} \mathrm{R} D^{g}$. Die wichtigsten Beispiele invarianter Relationen sind das Enthaltensein und die verschiedenen Grade der Vertauschbarkeit: $C \leqq D, C \vee D, C \leqq \mathrm{~N} D, C \leqq \mathrm{C} D$.

Satz 6. Es seien $C_{1}, \cdots, C_{k}$ Untergruppen von $G$. Für jedes einzelne $\kappa$ gelte wenigstens eine der beiden Beziehungen $A \leqq \mathrm{~N} C_{\kappa}, B \leqq \mathrm{~N} C_{\kappa}$. Ferner 
seien $\mathbf{R}_{\mathbf{1}}, \cdots, \mathbf{R}_{k}$ invariante Relationen zwischen Untergruppen von $G$. Schließlich sei $H$ eine Untergruppe von $G$, welche alle Relationen $H \mathbf{R}_{\kappa} C_{\kappa}$ erfüllt $(\kappa=1, \cdots, k)$, und es gebe keine von $H$ verschiedene Konjugierte $H^{g}$, welche ebenfalls alle diese Relationen erfüllt. Dann ist der Normalisator von $H$ in $G$ faktorisiert.

Beweis. Sei $a \in A, b \in B$. Wir haben $H^{a} \mathrm{R}_{\kappa} C_{\kappa}$, falls $A \leqq \mathrm{~N} C_{\kappa}$ ist; im andern Fall haben wir $H^{b} \mathbf{R}_{\kappa} C_{\kappa}$. Wenn also $H^{a}=H^{b}$ ist, so erfüllt diese Konjugierte von $H$ alle $k$ Relationen, daher stimmt sie mit $H$ überein, es ist $H^{a}=H$. Nach Hilfssatz 5 ist $\mathrm{N} H$ faktorisiert.

Wir erwähnen einen handlichen Sonderfall:

Hilfssatz 7. Sei $A_{*} \leqq\left|A, B_{*} \leqq\right| B$. Dann ist der Normalisator des Erzeugnisses $\left\{A_{*}, B_{*}\right\}$ faktorisiert.

Beweis. Wir können in Satz $6 C_{1}=A_{*}, C_{2}=B_{*}, H=\left\{A_{*}, B_{*}\right\}$ setzen und für $\mathbf{R}_{\mathbf{1}}, \mathbf{R}_{\mathbf{2}}$ die Relation $\geqq$ wählen.

\section{Hallgruppen}

Dem im nächsten Abschnitt zu erbringenden Beweis des Satzes 2 schicken wir einige Bemerkungen über die Hallgruppen $H$ einer endlichen Gruppe $G$ voraus; das sind diejenigen Untergruppen $H$ von $G$, deren Ordnung $|H|$ zu ihrem Index $|G: H|$ teilerfremd ist.

Hilfssatz 8. Sei $H$ eine nilpotente Hallgruppe von $G$. Sei U eine Untergruppe von $G$, deren Ordnung $|U|$ ein Teiler von $|H|$ ist. Dann gibt es in $G$ ein Element $g$ derart, daß $U^{g} \leqq H$ ist.

Das ist in [6] bewiesen worden.

Hilfssatz 9. Sei $H$ eine nilpotente Hallgruppe von $G$, aber nicht eine Sylowgruppe von $G$. Jede Sylowgruppe von $H$ habe in $G$ den Normalisator $H$. Dann enthält $G$ einen zu $H$ komplementären Normalteiler $N$, für welchen also $H N=G$ und $H \cap N=1$ ist.

Beweis. Sind $x, y$ zwei Elemente einer Sylowgruppe von $H$, welche in $G$ konjugiert sind, so sind sie schon in $H$ konjugiert. Denn $x$ und $y$ liegen beide im Zentralisator einer von $P$ verschiedenen Sylowgruppe $Q$ von $H$, und $Q$ ist Sylowgruppe von $G$; daher sind $x$ und $y$ nach einem bekannten Beweis von Burnside [1, p. 155] in $\mathrm{N} Q$ konjugiert.

Wenn man also für irgend eine Untergruppe $P_{1}$ von $P$ die von D. G. HIGMAN eingeführte Fokalgruppe $P_{2}$ bildet, welche von den in $P_{1}$ gelegenen Kommutatoren $x \circ g$ erzeugt wird $\left(x \in P_{1}, g \in G\right)$, so ist $P_{2} \leqq P_{1} \circ P$. Daher endet die Reihe der iterierten Fokalgruppen von $P$ mit 1; das heißt: $P$ ist hyperfokal im Sinn von Higman. Wenn alle Sylowgruppen einer Hallgruppe $H$ von $G$ hyperfokal sind, so ist $H$ hyperfokal [4, Theorem 4.6]. Ist eine 
Hallgruppe von $G$ hyperfokal, so enthält $G$ einen zu $H$ komplementären Normalteiler [4, Theorem 4.3].

Hilfssatz 10. Es sei $G=A B, A_{*} \leqq\left|A, B_{*} \leqq\right| B$. Dann gilt entweder für kein Paar oder für jedes Paar $g, h$ von Elementen von $G$ die Vertauschbarkeitsbeziehung $A_{*}^{g} B_{*}^{h}=B_{*}^{h} A_{*}^{g}$. Diese Beziehung gilt stets, wenn $G$ auflösbar ist und sowohl $A_{*}$ wie $B_{*}$ Hallgruppen von $G$ sind.

Beweis. Es seien $g, h$ beliebig gegebene Elemente von $G$. Wir zerlegen $h g^{-1}=b a^{-1}$ mit $a \in A, b \in B$. Dann sind je zwei benachbarte unter den folgenden Aussagen gleichwertig:

$$
A_{*}^{g} \vee B_{*}^{h}, \quad A_{*} \vee B_{*}^{h g^{-1}}, \quad A_{*} \vee B_{*}^{b a^{-1}}, \quad A_{*}^{a} \vee B_{*}^{b}, \quad A_{*} \vee B_{*} .
$$

Daher gilt $A_{*}^{g} \vee B_{*}^{h}$ entweder für kein oder jedes Paar $g, h$. Wenn $G$ auflösbar ist und $A_{*}, B_{*}$ Hallgruppen von $G$ sind, so gibt es nach P. HALL [3, p. 318] wenigstens ein vertauschbares Paar von Konjugierten zu $A_{*}$ und $B_{*}$ (und zwar genau eins in jedem Sylow-System von $G$ ).

\section{Beweis des Satzes 2}

Nach Hilfssatz 10 genügt es, folgendes zu beweisen:

Satz 2'. Eine endliche Gruppe, die das Produkt zweier nilpotenter Untergruppen mit teilerfremden Ordnungen ist, ist auflösbar. Jede Sylowgruppe des. ersten Faktors ist mit jeder Sylowgruppe des zweiten Faktors vertauschbar.

Diese Fassung ist bequem für den indirekten Beweis. Wir nehmen an, $G$ sei ein Gegenbeispiel minimaler Ordnung: Sei

$$
\begin{gathered}
G=A B, \quad(|A|,|B|)=1, \\
A=P_{1} \times \cdots \times P_{s}, \quad B=Q_{1} \times \cdots \times Q_{t} ;
\end{gathered}
$$

$P_{1}, \cdots, P_{r}$ seien die Sylowgruppen von $A ; Q_{1}, \cdots, Q_{t}$ die von $B ; G$ sei nicht auflösbar (aus der Auflösbarkeit würde nach Hilfssatz $10 P_{\sigma} \vee Q_{r}$ folgen). Wir leiten aus diesen Annahmen einen Widerspruch ab, indem wir der Reihe nach die folgenden Aussagen beweisen.

(a) $G$ ist einfach. Sei $1<N \leqq \mid G$. Die Faktorgruppe $G / N$ genügt den Voraussetzungen von Satz $2^{\prime}$, also ist sie auflösbar. Da $G$ nicht auflösbar ist, ist $N$ nicht auflösbar. Wir betrachten $A N$. Diese Gruppe ist nach Hilfssatz 4 faktorisiert und erfüllt daher die Voraussetzungen des Satzes $2^{\prime}$. Ferner ist $|A N| \leqq|G|$ und $A N$ nicht auflösbar, da $N$ nicht auflösbar ist. Wegen der Minimaleigenschaft von $|G|$ ist $|A N|=|G|$, also $A N=G$. Daher ist der Index $|G: N|=|A: A \cap N|$ ein Teiler von $|A|$; ebenso ist er ein Teiler von $|B|$. Da $|A|$ und $|B|$ teilerfremd sind, folgt $|G: N|=1$, $N=G$. Damit ist $G$ als einfach nachgewiesen.

(b) Es ist $s \geqq 2$ und $t \geqq 2$. Sonst sei etwa $s \leqq 1$. Dann ist $|G: B|$ eine Primzahlpotenz. Wir wählen ein $b \in \mathbf{Z} B, b \neq 1$. Dann ist $|G: \mathbf{C} b|$ 
eine Primzahlpotenz. Das widerspricht, nach einem bekannten Satz von Burnside [1, p. 322], der Einfachheit von $G$.

(c) Es sei $A_{*} \leqq P_{\sigma}, B_{*} \leqq Q_{\tau}, 1<\left\{A_{*}, B_{*}\right\}=H<G$. Dann enthält $H$ einen Normalteiler $N>1$, der in $P_{\sigma}$ oder in $Q_{\tau}$ liegt. $\quad$ Da $A_{*} \leqq \mid P_{\sigma}$ und $P_{\sigma}$ eine Sylowgruppe der nilpotenten Gruppe $A$ ist, haben wir $A_{*} \leqq A$; ebenso $B_{*} \leqq \mid B$. Wir setzen $\mathbf{N} H=\bar{G}$. Nach Hilfssatz 7 ist $\bar{G}$ faktorisiert: $\bar{G}=\bar{A} \bar{B}$ mit $\bar{A}=A \cap \bar{G}$ usw.; also erfüllt $\bar{G}$ die Voraussetzungen von Satz $2^{\prime}$. Ferner ist $\bar{G}<G$, denn $\bar{G}$ enthält den Normalteiler $H$, und $G$ ist einfach. Also gilt Satz $2^{\prime}$ für $\bar{G}$. Danach ist $\bar{G}$ auflösbar, und die beiden Sylowgruppen $\bar{P}_{\sigma}=P_{\sigma} \cap \bar{G}$ und $\bar{Q}_{\tau}=Q_{\tau} \cap \bar{G}$ sind vertauschbar. Es ist $H \leqq \bar{P}_{\sigma} \bar{Q}_{\tau}$. Als Normalteiler der auflösbaren Gruppe $\bar{G}$ ist auch $H$ auflösbar. Wir wählen in $H$ einen Normalteiler $N \neq 1$, der eine möglichst große Primzahlpotenz als Ordnung hat. Dann ist $N$ charakteristisch in $H$, daher normal in $\bar{G}$ und erst recht in $\bar{P}_{\sigma} \bar{Q}_{\tau}$. Als eine Gruppe von Primzahlpotenzordnung liegt also $N$ entweder in $\bar{P}_{\sigma}$ oder in $\bar{Q}_{\tau}$. Daher liegt $N$ in $P_{\sigma}$ oder in $Q_{\tau}$, wie behauptet.

(d) Für je zwei Indizes $\sigma, \tau$ ist $\left\{P_{\sigma}, Q_{\tau}\right\}=G$. Nehmen wir $\left\{P_{\sigma}, Q_{\tau}\right\}=H<G$ an. Nach (c) gibt es ein $N$ mit $1<N \leqq \mid\left\{P_{\sigma}, Q_{\tau}\right\}$ und etwa $N \leqq P_{\sigma}$. Es ist dann $N \leqq A$, und unter Benutzung der Einfachheit von $G$ erhalten wir den Widerspruch

$$
G=Q_{\tau}^{G}=Q_{\tau}^{B A}=Q_{\tau}^{A} \leqq\left\{A, Q_{\tau}\right\} \leqq \mathrm{N} N<G .
$$

(e) Ist $1 \neq a \in \mathbf{Z} A$, so ist $\left\{a, Q_{\tau}\right\}=G$ für jedes $\tau$. Zum Beweis können wir ohne Beschränkung der Allgemeinheit annehmen, daß $a$ Primzahlordnung hat (sonst ersetzen wir $a$ durch eine Potenz von $a$ ). Dann liegt $a$ in einer der Sylowgruppen von $A$, etwa $a \in P_{\sigma}$. Nehmen wir an, entgegen unserer Behauptung sei $\left\{a, Q_{\tau}\right\}<G$. Dann gibt es nach (c) eine Gruppe $N$ mit $1<N \leqq \mid\left\{a, Q_{r}\right\}$, für welche entweder $N \leqq P_{\sigma}$ oder $N \leqq Q_{\tau}$ gilt. Im ersten Fall können wir nach (b) einen Index $\rho \neq \sigma$ wählen und erhalten den Widerspruch $G=\left\{P_{\rho}, Q_{\tau}\right\} \leqq \mathrm{N} N<G$. Im zweiten Fall haben wir $N \leqq \mid B$ und erhalten den Widerspruch

$$
G=\{a\}^{G}=\{a\}^{A B}=\{a\}^{B} \leqq\{a, B\} \leqq \mathrm{N} N<G .
$$

(f) Ist $1 \neq a \in \mathbf{Z} A$ und $1 \neq b \in \mathbf{Z} B$, so ist $\{a, b\}=G$. Wir können annehmen, daß $a$ und $b$ Primzahlordnungen haben. Dann ist etwa $a \epsilon P_{\sigma}, b \in Q_{\rho}$. Nach (c) gibt es eine Gruppe $N$ mit $1<N \leqq \mid\{a, b\}$, welche entweder in $P_{\sigma}$ oder in $Q_{\rho}$ liegt; sagen wir in $Q_{\rho}$. Wir wählen $Q_{\tau} \neq Q_{\rho}$. Dann führt (e) auf den Widerspruch $G=\left\{a, Q_{\tau}\right\} \leqq \mathrm{N} N<G$.

(g) Ist $1 \neq a \in \mathbf{Z} A$, so ist $\mathrm{C} a=A$. Andernfalls ist $\mathrm{C} a>A$. Nach Hilfssatz 4 ist $C_{a}$ faktorisiert, enthält also ein Element $b \neq 1$ aus einem passenden $Q_{\rho}$. Wir wählen $Q_{\tau} \neq Q_{\rho}$ und erhalten mittels (e) den Widerspruch $G=\left\{a, Q_{\tau}\right\} \leqq \mathrm{C} b<G$. 
(h) Ist g $\epsilon G$ und $P_{\sigma} \leqq A^{g}$ für ein $\sigma$, so ist $A^{g}=A$. Da $A^{g}$ nur eine Untergruppe der Ordnung $\left|P_{\sigma}\right|$ enthält, ist $P_{\sigma}^{g}=P_{\sigma}$. Nach (g) haben wir $A=\mathrm{CZ} P_{\sigma}$. Hieraus folgt

$$
A^{g}=\left(\mathrm{CZ} P_{\sigma}\right)^{g}=\mathrm{CZ}\left(P_{\sigma}^{g}\right)=\mathrm{CZ} P_{\sigma}=A .
$$

(i) Ist $g \in G$ und $A \cap \mathbf{Z} A^{g}>1$, so ist $A^{g}=A$. Zum Beweis wählen wir in $A \cap \mathrm{Z} A^{g}$ ein Element $a$ von Primzahlordnung, etwa $a \in P_{\rho}$. Es ist $a \in P_{\rho} \cap \mathrm{Z} P_{\rho}^{g}$ und nach (g) $\mathrm{C} a=A^{g}$. Wir wählen $P_{\sigma} \neq P_{\rho}$ und finden $P_{\sigma} \leqq \mathrm{C} a=A^{g}$. Nach (h) folgt nun $A^{g}=A$.

(j) Ist $\mathrm{Z} A \leqq \bar{G} \leqq G$ und $\bar{P}_{\sigma}=P_{\sigma} \cap \bar{G}$, so ist $\bar{P}_{\sigma}$ eine Sylowgruppe von $\bar{G}$. Zum Beweis wählen wir eine $\bar{P}_{\sigma}$ enthaltende Sylowgruppe $P$ von $\bar{G}$. Wir haben dann mit passendem $g \in G$

$$
1<\mathrm{Z} P_{\sigma} \leqq P_{\sigma} \cap \bar{G}=\bar{P}_{\sigma} \leqq P \leqq P_{\sigma}^{g} \leqq A^{g} .
$$

Nach (i) folgt weiter $A^{g}=A$, hieraus $P_{\sigma}^{g}=P_{\sigma}$. Nun finden wir

$$
P \leqq P_{\sigma}, \quad \bar{P}_{\sigma} \leqq P \leqq P_{\sigma} \cap \bar{G}=\bar{P}_{\sigma}, \quad \bar{P}_{\sigma}=P .
$$

(k) Es sei $\mathrm{Z} A \leqq \bar{G} \leqq G$, und es werde $P_{\sigma} \cap \bar{G}=\bar{P}_{\sigma}, A \cap \bar{G}=\bar{A}$ gesetzt. Ist dann $\bar{G} \cap \mathbf{N} \bar{P}_{\sigma}=\bar{A}$ für alle $\sigma$, so ist $\bar{G} \leqq A$. Es ist $\bar{A}=\bar{P}_{1} \times \cdots \times \bar{P}_{s}$, also ist $\bar{A}$ nach (j) eine nilpotente Hallgruppe von $\bar{G}$ mit den Sylowgruppen $\bar{P}_{\sigma}$. Weil stets $\bar{G} \cap \mathbf{N} \bar{P}_{\sigma}=\bar{A}$ ist, gibt es nach Hilfssatz 9 in $\bar{G}$ einen zu $\bar{A}$ komplementären Normalteiler $N$; seine Ordnung ist ein Teiler von $|B|$. Nach Hilfssatz 8 gibt es ein $g \in G$ derart, daß $N^{g} \leqq B$ ist. Zerlegen wir $g=a b, a \epsilon A, b \in B$, so haben wir $N^{a b} \leqq B$, daher $N^{a} \leqq B$. Es ist also $\mathrm{Z} B \leqq \mathbf{N} N^{a}$. Andererseits ist wegen $\mathbf{Z} A \leqq \bar{G} \leqq \mathbf{N} N$ auch

$$
\mathrm{Z} A=\mathbf{Z} A^{a} \leqq \mathrm{~N} N^{a} .
$$

Aus (f) wissen wir, daß $\{Z A, Z B\}=G$ ist. Also haben wir $N^{a} \leqq \mid G$. Es ist $|N|$ ein Teiler von $|B|$, also $N<G$. Wegen der Einfachheit von $G$ ergibt sich $N=1$. Da $N$ in $\bar{G}$ komplementär zu $\bar{A}$ ist, folgt $\bar{G}=$ $\bar{A} \leqq A$, wie behauptet.

(l) Von nun an setzen wir ständig $A^{*}=A \cap \mathrm{N} B, B^{*}=B \cap \mathrm{N} A$. Wir behaupten: $A^{*}$ enthält zu jeder Primzahl $p$ höchstens eine Untergruppe der Ordnung $p$. Die Ähnlichkeitstransformation mit einem Element aus $A^{*}$ führt $\mathbf{Z} B$ in sich über. Nach (g), angewandt auf $B$ statt $A$, stellen die so entstehenden Automorphismen von $\mathrm{Z} B$ die Gruppe $A^{*}$ treu dar und sind überdies regulär, d.h. jeder einzelne dieser Automorphismen läßt entweder kein oder jedes Element $\neq 1$ von $Z B$ fest. Eine reguläre Automorphismengruppe enthält nach BuRnside [1, p. 335] keine Untergruppe vom Typus $(p, p)$. Also enthält auch $A^{*}$ keine solche Untergruppe. Da $A^{*}$ außerdem nilpotent ist, enthält $A^{*}$ höchstens eine Untergruppe der Ordnung $p$. 
(m) Ist g $\in G$ und $A^{*} \cap A^{g}>1$, so ist $A^{g}=A$. Zum Beweis wählen wir ein Element $a$ von Primzahlordnung in $A^{*} \cap A^{g}$, etwa $a \in P_{\rho}$, und dazu eine Gruppe $P_{\sigma} \neq P_{\rho}$. Die Gruppe $H=\{a\}$ erfüllt die invarianten Relationen $H \leqq \mathrm{~N} B, H \leqq A$. Nach (l) erfüllt keine von $H$ verschiedene Konjugierte $H^{g}$ beide Relationen. Nach Satz 6 ist die Gruppe $\bar{G}=\mathrm{N} H$ faktorisiert:

$$
\bar{G}=\left(\bar{P}_{1} \times \cdots \times \bar{P}_{s}\right)\left(\bar{Q}_{1} \times \cdots \times \bar{Q}_{t}\right),
$$

und für den ausgezeichneten Index $\sigma$ ist $\bar{P}_{\sigma}=P_{\sigma} \cap \bar{G}=P_{\sigma}$ wegen $P_{\sigma} \epsilon \mathrm{CH}$. Da $G$ einfach ist, ist $\bar{G}<G$, daher $\bar{G}$ auflösbar; und wenn wir $\tau$ beliebig wählen, $P_{\sigma} \vee \bar{Q}_{\tau}$. Wir wählen in $P_{\sigma} \bar{Q}_{\tau}$ einen Normalteiler $N$ von Primzahlpotenzordnung; es ist $N \leqq \mid P_{\sigma}$ oder $N \leqq \mid \bar{Q}_{\tau}$. Die Annahme $N \leqq \bar{Q}_{r}$ führt auf einen Widerspruch, wenn wir ein $Q_{\rho} \neq Q_{r}$ betrachten: $G=\left\{P_{\sigma}, Q_{\rho}\right\} \leqq \mathrm{N} N<G$. Es ist also $N \leqq \mid P_{\sigma}$, daher gibt es ein $n \in N$ mit $1 \neq n \in N \cap \mathrm{Z} P_{\sigma} \leqq \mathrm{Z} A$. Nun ist $\{n\}^{\overline{\bar{\theta}}_{\tau}} \leqq N \leqq P_{\sigma} \leqq A$. Nach (i) folgt $\bar{Q}_{\tau} \leqq \mathrm{N} A$. Da dies für alle $\tau$ gilt, ist $\bar{G} \cap B \leqq \mathrm{~N} A$. Da $\bar{G}$ faktorisiert und $\bar{G} \cap A \leqq \mathrm{~N} A$ ist, ist $\bar{G} \leqq \mathrm{~N} A$. Nach Konstruktion ist $a \in A^{g}$, also ist $\mathrm{Z} A^{g} \leqq \mathrm{~N}\{a\}=\bar{G} \leqq \mathrm{~N} A$. Durch Betrachtung der Ordnungen folgt nun $\mathrm{Z} A^{g} \leqq A$, und hieraus folgt nach (i), daß $A^{g}=A$ ist, wie behauptet.

(n) Ist $a \in A$ und $B^{*} \mathrm{n} \mathrm{C} a>1$, so ist $a \in A^{*}$. Es ist

$$
1<B^{*} \cap \mathrm{C} a=\left(B^{*} \cap \mathrm{C} a\right)^{a} \leqq B^{*} \cap B^{*^{a}} \leqq B^{*} \cap B^{a} .
$$

Nach (m), angewandt auf $B$ statt $A$, ergibt sich $B^{a}=B, a \in A^{*}$.

(o) Ist $\mathrm{Z} A \leqq \bar{G} \leqq A$, so gibt es ein $a \in A$ derart, daß $B^{*} \cap \bar{G}^{a}>1$ ist. Nach (j) ist der Durchschnitt $A \cap \bar{G}=\bar{A}$ eine nilpotente Hallgruppe von $\bar{G}$ mit den Sylowgruppen $\bar{P}_{\sigma}=P_{\sigma} \cap \bar{G}$. Da wir $\bar{G} \$ A$ vorausgesetzt haben, kann nach (k) nicht stets $\bar{G} \cap \mathbf{N} \bar{P}_{\sigma}=\bar{A}$ sein; es gibt ein $\sigma$ mit $\bar{G} \cap \mathrm{N} \bar{P}_{\sigma}>\bar{A}$. Da $\bar{A}$ eine zu den Primteilern von $|A|$ gehörige Hallgruppe von $\bar{G}$ ist, gibt es einen Primteiler $q$ von $|B|$, der in $\left|\bar{G} \cap \mathbf{N} \bar{P}_{\sigma}\right|$ aufgeht. Wir wählen ein Element $x$ der Ordnung $q$ in $\bar{G} \cap \mathrm{N} \bar{P}_{\sigma}$. Dann wird $\mathrm{Z} P_{\sigma} \leqq \bar{P}_{\sigma}=\bar{P}_{\sigma}^{x} \leqq A^{x}$; nach (i) folgt $x \in \mathrm{N} A$. Ferner gibt es nach Hilfssatz 8 ein Element in $G$, welches $x$ nach $B$ transformiert, und wegen $G=A B$ kann man dieses transformierende Element in $A$ wählen: es gibt ein $a \epsilon A$ mit $x^{a} \in B$. Wegen $x \in \mathbf{N} A$ haben wir $x^{a} \in \mathbf{N} A$, und wegen $x \in \bar{G}$ ist $x^{a} \in \bar{G}^{a}$. Insgesamt haben wir $x^{a} \in B \cap \mathbf{N} A \cap \bar{G}^{a}$, daher ist $B^{*} \cap \bar{G}^{a}>1$.

(p) Ist g $\in G$ und $A \neq A^{g}$, so ist $A \cap A^{g}=1$. Wir setzen $\bar{G}=\left\{Z A, Z A^{g}\right\}$. Dann ist $\bar{G} \leqq \mathrm{C}\left(A \cap A^{g}\right)$. Nach (i) ist ferner $\mathbf{Z} A^{g} \leqq A$, also ist erst recht $\bar{G} \equiv A$. Daher gibt es nach (o) ein $a \in A$ derart, daß $B^{*} \cap \bar{G}^{a}>1$ ist. Aus $\bar{G}^{a} \leqq \mathrm{C}\left(A \cap A^{g a}\right)$ folgt nun $B^{*} \cap \mathrm{C}\left(A \cap A^{g a}\right)>1$ und hieraus nach (n) weiter $A \cap A^{g a} \leqq A^{*} \cap A^{g a}$. Wegen $A^{g} \neq A$ ist ferner $A^{g a} \neq A$; nach (m) ist also $A^{*} \cap A^{g a}=1$. Daher ist $A \cap A^{g a}=1$, also $A \cap A^{g}=1$.

(q) Nun kommen wir zu dem Widerspruch, der den Beweis beendet: $\mathrm{Da}|A|$ und $|B|$ teilerfremd und $>1$ sind, ist $|A| \neq|B|$. Sei etwa 
$|B|<|A|$. Dann ist $|G|=|A| \cdot|B|<|A|^{2}$. Andererseits sind nach (p) alle $|A|^{2}$ Elemente des Komplexes $A A^{g}$ voneinander verschieden, wenn $g$ irgend ein nicht in $\mathrm{N} A$ gelegenes Element von $G$ bedeutet.

\section{Literatur}

1. W. Bunnside, Theory of groups of finite order, $2^{\text {nd }}$ ed., Cambridge, 1911; New York, 1955.

2. P. Hall, A characteristic property of soluble groups, J. London Math. Soc., vol. 12 (1937), pp. 198-200.

3. - - On the Sylow systems of a soluble group, Proc. London Math. Soc. (2), vol. 43 (1937), pp. 316-323.

4. D. G. Higman, Focal series in finite groups, Canadian J. Math., vol. 5 (1953), pp. 477-497.

5. H. Wielandt, Über das Produkt paarweise vertauschbarer nilpotenter Gruppen, Math. Zeitschr., vol. 55 (1951), pp. 1-7.

6. ——, Zum Satz von Sylow, Math. Zeitschr., vol. 60 (1954), pp. 407-408.

\section{UNIVERSITÄT}

TÜBIngen, Deutschland 